\title{
Annealing Effects on the Phase and Electronic Structure Evolutions of SiO Film by Electron Energy Loss Spectroscopy
}

\author{
Juan Wang ${ }^{*}$, X. F. Wang ${ }^{*}$, A. Meldrum ${ }^{* *}$, and Quan $\mathrm{Li}^{*}$ \\ * Department of Physics, the Chinese University of Hong Kong, Shatin, New Territory, Hong \\ Kong \\ ${ }^{* *}$ Department of Physics, University of Alberta, Edmonton, AB, T6G 2J1, Canada
}

Composite films consisting of Si nanoclusters embedded in silicon oxide (also known as silicon rich oxide (SRO)) have attracted much attention due to their potential technological importance in the Si-based optoelectronic industry. The annealing process significantly affects the luminescence of the SRO films, causing major changes in the integrated luminescence intensity and the peak wavelength. The effect of annealing has been generally summarized as the generation of $\mathrm{Si}$ nanocrystals hosted in the $\mathrm{SiO}_{2}$ matrix, followed by a nanocrystal growth process as the annealing temperature increases [1]. The luminescence has always been ascribed to the radiative recombination process in the Si nanocrystals or at the interface [2]. However, it has been found that even the low temperature (well below what would be expected to generate the Si nanocrystals) annealed SRO films give luminescence peak(s) in the visible range [3]. As the optical properties of the films are determined by the microstructure/electronic structure of the material, it is important to understand the films' structure change as a function of the annealing temperature.

In the present work [4], we have carried out a systematic study on the microstructure and electronic structure evolution of the as-deposited SRO film and the films annealed at temperatures ranging from $300-1100^{\circ} \mathrm{C}$, using combined transmission electron microscopy and electron energy loss (EEL) spectroscopy related techniques. We have found that the asdeposited SRO film is basically a single phase $\mathrm{SiO}_{1.0}$, as suggested by its electronic structure characteristics disclosed by valence electron energy loss spectrum. Such single phase undergoes a continuous but incomplete phase decomposition to $\mathrm{Si}$ and $\mathrm{SiO}_{2}$, indicating that the matrix of the $\mathrm{Si}$ clusters is not phase pure $\mathrm{SiO}_{2}$, but a mixture of $\mathrm{SiO}_{2}$ and $\mathrm{SiO}$ (i.e., $\mathrm{SiO}_{x}$ ). The resulted $\mathrm{Si}$ phase first appears as small amorphous clusters, which continue to grow to larger sizes at higher annealing temperatures, but only crystallize at a critical temperature of $\sim 800-900^{\circ} \mathrm{C}$. Such cluster/matrix configuration of the annealed $\mathrm{SiO}$ films is also consistent with the appearance of the interfacial plasmon in the EEL spectrum and its oscillation strength change with the annealing temperature. An interesting correlation between the films' phase/electronic structure evolution and the trend of their photoluminescence property change is identified.

\section{References}

[1] U. Kahler and H. Hofmeister, Appl. Phys. A 74, (2002) 13.

[2] G. G. Qin, Mater. Res. Bull. 33, (1998) 1857.

[3] O. Hanaizumi, K. Ono and Y. Ogawa, Appl. Phys. Lett. 82, (2003) 538.

[4] This work is supported by a grant of the Research Grant Council of Hong Kong SAR under project No. CUHK 402105. The U-Alberta group is funded by iCORE, the PRF, and NSERC. 


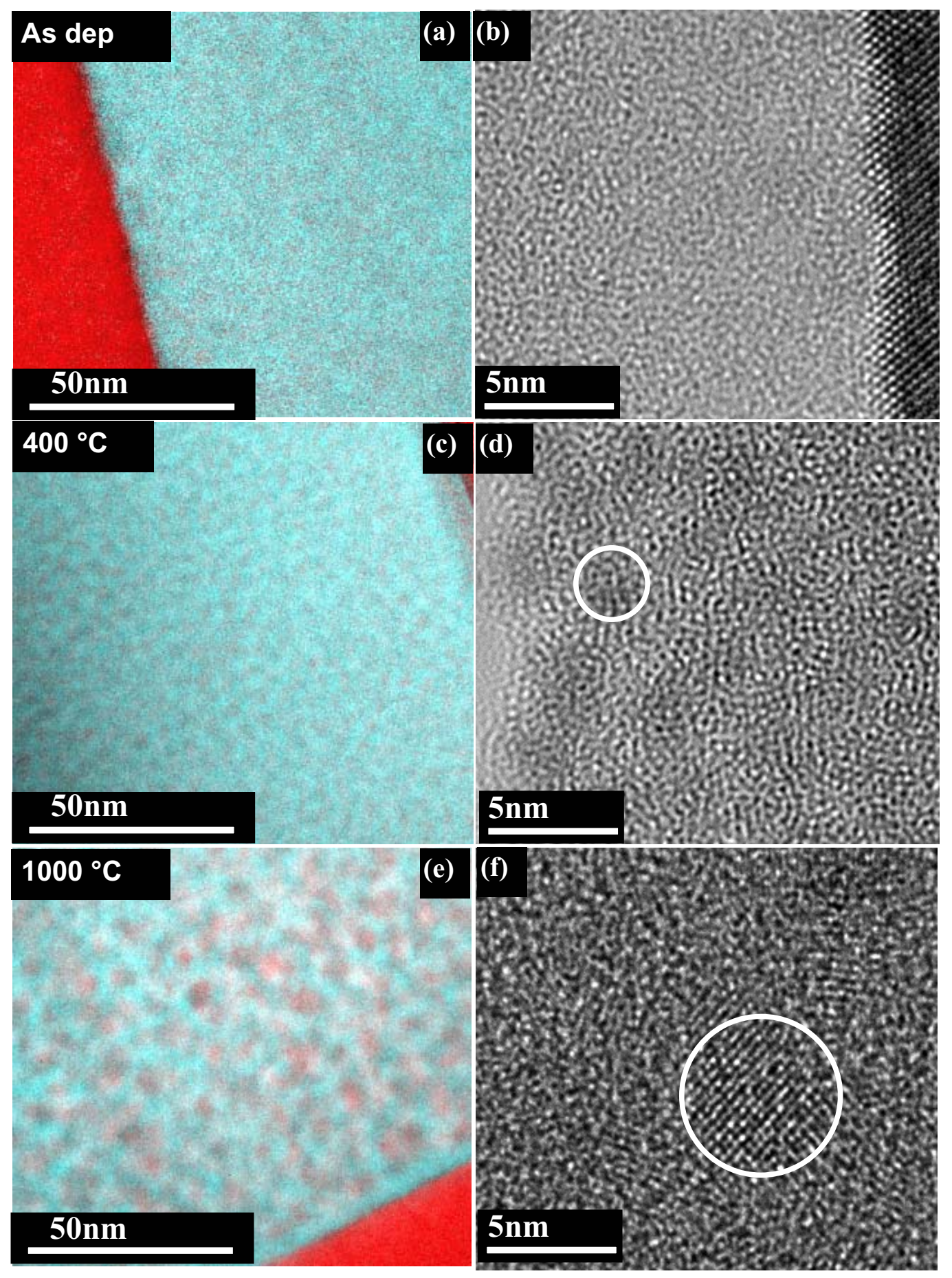

FIG. 1. Selected elemental maps and high resolution images of the SRO films annealed in different temperature. (a), (c), (e): The overlapped elemental maps of the as-deposited film and those annealed at 400 and $1000{ }^{\circ} \mathrm{C}$, with $\mathrm{Si}$ in red and $\mathrm{O}$ in blue; (b), (d), (f): The corresponding high resolution images. 